\title{
Virulence Factors and Molecular Epidemiology of Pyogenic Liver Abscess Causing Multidrug Resistant Klebsiella pneumoniae in Wenzhou, China
}

\section{Zhongyong Wang}

the First Affiliated Hospital of Wenzhou Medical University

\section{Siqin Zhang}

the First Affiliated Hospital of Wenzhou Medical University

\section{Na Huang}

the First Affiliated Hospital of Wenzhou Medical University

Shixing Liu

the First Affiliated Hospital of Wenzhou Medical University

YeXu

the First Affiliated Hospital of Wenzhou Medical University

Yajie Zhao

Wenzhou Medical University

Jianming Cao

Wenzhou Medical University

Tieli Zhou ( $\nabla$ wyztli@163.com )

Wenzhou Medical University First Affiliated Hospital https://orcid.org/0000-0002-2171-4710

\section{Research article}

Keywords: pyogenic liver abscess, multidrug resistance, Klebsiella pneumoniae, virulence factors, molecular epidemiology

Posted Date: July 17 th, 2020

DOI: https://doi.org/10.21203/rs.3.rs-42660/v1

License: @ (i) This work is licensed under a Creative Commons Attribution 4.0 International License. Read Full License 


\section{Abstract}

Background: To date, little is known about virulence characteristics of pyogenic liver abscess (PLA) causing multidrug resistant (MDR) Klebsiella pneumoniae (K. pneumoniae). It may be these strains are rare. The aim of this study was to analyze the virulence and molecular epidemiology of 12 MDR strains from 163 PLA cases in a tertiary teaching hospital from the perspective of clinical characteristics, virulence phenotypes and genotypes.

Results: The virulence phenotypes of the twelve PLA-causing MDR K. pneumoniae were similar or even more obvious than those of sensitive hypervirulent Klebsiella pneumoniae control strains. These MDR strains were mainly non-K1/K2 serotypes and carried multiple virulence genes. Multilocus sequence typing (MLST) revealed the PLA-causing MDR strains had low clone correlation.

Conclusions: This study was the first analysis on virulence factors of PLA-causing MDR strains. The virulent factors exhibited the coexistence of hypervirulence and multidrug resistance in PLA-causing MDR K. pneumoniae strains, and these MDR strains were mainly polyclonal spread and there was firstly found one ST11 carbapenem-resistant hypervirulent strain in PLA.

\section{Background}

Klebsiella pneumoniae has emerged as a major Gram-negative bacterium for urinary tract infections, pneumonia, bacteremia, and intra-abdominal infections worldwide [1-2]. During the past three decades, K. pneumoniae has been divided into two largely non-overlapping populations. One is termed classic $K$. pneumoniae (cKP), which is considered as the pathogen causing hospital-acquired infections in immunocompromised patients, notorious for acquiring antimicrobial resistance [1-3]. The other is hypervirulent K. pneumoniae (hvKP). In contrast to cKP, the emerging variant, which was first reported in Taiwan in 1986 , is hypervirulent for causing severe invasive community-acquired infections and disseminates infections among immunocompetent individuals, and it exhibits hypervirulent phenotypes and genotypes, which is susceptible to conventional antimicrobial agents except for being intrinsically resistant to ampicillin [1-3].

Pyogenic liver abscess (PLA) is a potentially life-threatening suppurating infection of hepatic parenchyma disease worldwide $[2,4,5]$. K. pneumoniae has emerged as a predominant pathogen of PLA across Asian and European countries, as well as the United States, and there is no denying that $K$. pneumoniae induced pyogenic liver abscess (KP-PLA) remains a mortalityassociated serious clinical challenge [5-6]. Hypervirulent $K$. pneumoniaeinduced PLA usually occurs in young and healthy community individuals with cryptogenic abscess in which no source of infection could be identified, and then, migrates to distant sites, thereby leading to extrahepatic complications, such as endophthalmitis, meningitis, and necrotizing fasciitis [2, 5, 7]. Most of isolates from KP-PLA are susceptible to the majority of antimicrobials, and the antimicrobial resistance rates are less than 10\% [8]. Antibiotic resistance is a phenomenon associated primarily with cKP. Alarmingly, recent reports revealed the convergence of virulence and resistance in $K$. pneumoniae, most of these phenomena were commonly caused by plasmid mediated resistance traits and virulence genes transfer [9-10]. In our previous study [11], twelve multidrug resistant (MDR) $K$. pneumoniae were isolated from non-cryptogenic PLA and tended to be considered as cKP. Although, KP-PLA caused by antimicrobial-susceptible hypervirulent strains has been well reported, MDR K. pneumoniae isolates from KP-PLA are rare and have not been well identified, particularly in the virulence and molecular epidemiology in the literature $[2,7,12]$. Whether these MDR K. pneumoniae isolates were indeed typical CKP or combined with hypervirulence was unknown. Since the convergence of virulence and resistance in K. pneumoniae, as well as the challenge of KP-PLA itself, gaining knowledge of virulence and molecular epidemiological characteristics in PLA-causing multidrug resistant $K$. pneumoniae has become more urgent. The virulence of $K$. pneumoniae is closely related to the ability of aggression and defense, and hypervirulence can assist pathogen to resist host innate immunity and infect the host invasively with high pathogenicity $[1,13]$. In addition, hypervirulent strains usually possess thick capsular polysaccharide, anti-serum capacity and multiple virulence factors, including hypermucoviscosity, capsular serotype, virulence genes, and related clones [14, 15].

The purpose of the present study was to investigate the virulence factors and molecular epidemiology of PLA-causing multidrug resistant $K$. pneumoniae, by collecting strains over a 2-year period from KP-PLA patients in a tertiary teaching 
hospital, in order to provide significant insights for the development of further effective therapeutic strategies for clinical trials of KP-PLA.

\section{Results}

\section{Antimicrobial susceptibility testing}

Among 12 PLA-causing MDR strains, the resistance rates to cephalosporins (ceftriaxone, ceftazidime, cefepime), quinolones (ciprofloxacin), sulfamethoxazole/trimethoprim and nitrofurantoin were high (50\%-100\%). Thereinto, three strains were resistant to carbapenem, one of which was resistant to colistin, but these strains were more sensitive to aminoglycosides.

However, the 12 hypervirulent control strains were sensitive to all tested antibacterials with the exception for being intrinsically resistant to ampicillin (Table 1). 
Table 1

The MICs of PLA-causing multidrug resistant strains and control strains against commonly used antimicrobial agents

\begin{tabular}{|c|c|c|c|c|c|c|c|c|c|c|c|c|c|}
\hline \multirow{2}{*}{$\begin{array}{l}\text { Strain } \\
\text { numbers }\end{array}$} & \multicolumn{13}{|c|}{ MICs ( $\mu \mathrm{g} / \mathrm{mL}, \mathrm{mg} / \mathrm{L})$} \\
\hline & AMP & ATM & CRO & CAZ & FEP & IPM & CIP & LEV & GEN & TOB & SXT & NIT & COL \\
\hline FK3038 & $>64$ & 2 & $>64$ & 16 & 4 & $>64$ & $>8$ & 1 & 2 & 1 & $\begin{array}{l}< \\
0.25 / 4.75\end{array}$ & 128 & 0.5 \\
\hline FK3044 & 32 & 16 & $>64$ & $>64$ & 32 & 1 & 0.03 & 2 & 0.125 & 4 & $>16 / 304$ & 128 & 1 \\
\hline FK3068 & 32 & 16 & $>64$ & 16 & 32 & 0.5 & 0.03 & 2 & 0.5 & 4 & $>16 / 304$ & 128 & 0.25 \\
\hline FK3228 & 32 & $>64$ & $>64$ & $>64$ & $>32$ & 4 & 0.06 & $>16$ & 0.125 & 4 & $>16 / 304$ & 256 & 16 \\
\hline FK3347 & 32 & 2 & 4 & 8 & $>32$ & 1 & 0.5 & $>16$ & 0.5 & 4 & $>16 / 304$ & 256 & 0.25 \\
\hline FK3518 & $>64$ & 2 & $>64$ & 16 & 2 & 1 & $>8$ & 1 & 0.5 & 0.5 & $1 / 19$ & 256 & 0.25 \\
\hline FK3521 & $>64$ & 2 & $>64$ & 16 & 16 & 1 & $>8$ & 4 & 0.5 & $\begin{array}{l}<.25 \\
0.25\end{array}$ & $\begin{array}{l}< \\
0.25 / 4.75\end{array}$ & 128 & 0.25 \\
\hline FK3599 & 32 & 2 & 4 & 2 & 0.25 & 4 & 8 & 1 & 0.5 & $\begin{array}{l}<.25 \\
0.25\end{array}$ & $\begin{array}{l}< \\
0.25 / 4.75\end{array}$ & 256 & 0.25 \\
\hline FK4176 & $>64$ & 2 & 64 & 8 & 16 & 0.5 & 2 & 4 & 16 & 0.5 & $\begin{array}{l}< \\
0.25 / 4.75\end{array}$ & 128 & 0.5 \\
\hline FK4276 & $>64$ & 2 & $>64$ & 32 & 16 & 1 & 4 & 1 & 16 & $\begin{array}{l}<.25 \\
0.25\end{array}$ & $\begin{array}{l}< \\
0.25 / 4.75\end{array}$ & 32 & 1 \\
\hline FK4603 & 32 & $>64$ & 64 & 64 & $>32$ & 1 & 4 & $>16$ & 0.25 & 2 & $>16 / 304$ & 256 & 0.25 \\
\hline FK4737 & 32 & 32 & $>64$ & 32 & 8 & 2 & 4 & 4 & 0.5 & 4 & $>16 / 304$ & 128 & 0.125 \\
\hline FK3112 & 32 & 1 & $\begin{array}{l}< \\
0.03\end{array}$ & 0.125 & $\begin{array}{l}<.03 \\
0.03\end{array}$ & 0.125 & 0.03 & 2 & 0.25 & 1 & $0.5 / 9.5$ & 32 & 0.5 \\
\hline FK3262 & 32 & 0.5 & $\begin{array}{l}< \\
0.03\end{array}$ & 0.125 & $\begin{array}{l}<.03 \\
0.03\end{array}$ & 0.125 & 0.03 & 0.06 & 0.25 & 0.5 & $0.5 / 9.5$ & 32 & 0.25 \\
\hline FK3645 & $>64$ & 0.5 & $\begin{array}{l}< \\
0.03\end{array}$ & 0.125 & $\begin{array}{l}< \\
0.03\end{array}$ & 0.125 & 0.03 & 0.06 & 0.5 & 0.5 & $\begin{array}{l}< \\
0.25 / 4.75\end{array}$ & 32 & 1 \\
\hline FK3698 & 32 & 0.5 & $\begin{array}{l}< \\
0.03\end{array}$ & 0.25 & $\begin{array}{l}< \\
0.03\end{array}$ & 0.25 & 0.03 & 0.06 & 1 & $\begin{array}{l}< \\
0.25\end{array}$ & $\begin{array}{l}< \\
0.25 / 4.75\end{array}$ & 16 & 0.25 \\
\hline FK3736 & 32 & 0.25 & $\begin{array}{l}< \\
0.03\end{array}$ & 0.125 & $\begin{array}{l}< \\
0.03\end{array}$ & 0.25 & 0.03 & 1 & 0.5 & 0.5 & $\begin{array}{l}< \\
0.25 / 4.75\end{array}$ & 16 & 0.5 \\
\hline FK3818 & 32 & 1 & $\begin{array}{l}< \\
0.03\end{array}$ & 0.25 & $\begin{array}{l}< \\
0.03\end{array}$ & 0.25 & 0.5 & 0.25 & 0.25 & 0.5 & $\begin{array}{l}< \\
0.25 / 4.75\end{array}$ & 32 & 1 \\
\hline FK3837 & 32 & 1 & $\begin{array}{l}< \\
0.03\end{array}$ & 0.25 & $\begin{array}{l}< \\
0.03\end{array}$ & 0.25 & 0.03 & 0.06 & 0.25 & 0.5 & $\begin{array}{l}< \\
0.25 / 4.75\end{array}$ & 32 & 0.25 \\
\hline FK3914 & 32 & 0.5 & $\begin{array}{l}< \\
0.03\end{array}$ & 0.125 & $\begin{array}{l}< \\
0.03\end{array}$ & 0.25 & 0.03 & 0.06 & 0.25 & 0.5 & $\begin{array}{l}< \\
0.25 / 4.75\end{array}$ & 32 & 0.25 \\
\hline FK3953 & 32 & 0.5 & $\begin{array}{l}< \\
0.03\end{array}$ & 0.125 & $\begin{array}{l}< \\
0.03\end{array}$ & 0.25 & 0.03 & 0.5 & 0.5 & 0.5 & $\begin{array}{l}< \\
0.25 / 4.75\end{array}$ & 32 & 0.5 \\
\hline
\end{tabular}

MICs minimum inhibitory concentrations, PLA pyogenic liver abscess, AMP ampicillin, ATM aztreonam, CRO ceftriaxone, CAZ ceftazidime, FEP cefepime, IPM imipenem, CIP ciprofloxacin, LVX levofloxacin, GEN gentamicin, TOB tobramycin, SXT sulfamethoxazole/trimethoprim, NIT nitrofurantoin, COL colistin.

Strain numbers were underlined: multidrug resistant strains; strain numbers were bolded: hypervirulent control strains. gray shading: resistance, white shading: intermediate or susceptible 


\begin{tabular}{|c|c|c|c|c|c|c|c|c|c|c|c|c|c|}
\hline \multirow{2}{*}{$\begin{array}{l}\text { Strain } \\
\text { numbers }\end{array}$} & \multicolumn{13}{|c|}{ MICs ( $\mu \mathrm{g} / \mathrm{mL}, \mathrm{mg} / \mathrm{L})$} \\
\hline & AMP & ATM & CRO & CAZ & FEP & IPM & CIP & LEV & GEN & ТОВ & SXT & NIT & COL \\
\hline FK3992 & 32 & 0.5 & $\begin{array}{l}<.03 \\
0.03\end{array}$ & 0.125 & $\begin{array}{l}<.03 \\
0.03\end{array}$ & 0.25 & 0.008 & 0.25 & 0.25 & 0.5 & $\begin{array}{l}< \\
0.25 / 4.75\end{array}$ & 32 & 0.5 \\
\hline FK4081 & $>64$ & 0.5 & $\dot{0.03}$ & 0.125 & $\begin{array}{l}<.03 \\
0.03\end{array}$ & 0.125 & 0.03 & 0.06 & 0.25 & 0.5 & $\begin{array}{l}< \\
0.25 / 4.75\end{array}$ & 32 & 1 \\
\hline FK4578 & $>64$ & 0.5 & $\begin{array}{l}<.03 \\
0.03\end{array}$ & 0.125 & $\begin{array}{l}<.03 \\
0.03\end{array}$ & 0.25 & 0.03 & 0.06 & 0.25 & $\begin{array}{l}<.25 \\
0.25\end{array}$ & $\begin{array}{l}< \\
0.25 / 4.75\end{array}$ & 16 & 1 \\
\hline \multicolumn{14}{|c|}{$\begin{array}{l}\text { MICs minimum inhibitory concentrations, PLA pyogenic liver abscess, AMP ampicillin, ATM aztreonam, CRO ceftriaxone, } \\
\text { CAZ ceftazidime, FEP cefepime, IPM imipenem, CIP ciprofloxacin, LVX levofloxacin, GEN gentamicin, TOB tobramycin, SXT } \\
\text { sulfamethoxazole/trimethoprim, NIT nitrofurantoin, COL colistin. }\end{array}$} \\
\hline
\end{tabular}

\section{Growth curves}

The growth ability of MDR strains was comparable with that of hypervirulent control strains and the standard strain ATCC 700603 (Fig. 1).

\section{String test and quantification of capsule}

String test disclosed that two (2/12, 16.6\%) MDR strains had hypermucoviscosity, while five (5/12, 41.7\%) strains had hypermucoviscosity among the hypervirulent control strains. Quantification of capsule further revealed the capsular polysaccharide content of MDR strains was significantly lower than that of hypervirulent control strains, but significantly higher than ATCC 700603, and the differences were statistically significant $(P<0.05)$ (Fig. 2).

\section{Susceptibility to serum killing}

All MDR strains and hypervirulent control strains isolated from KP-PLA were susceptible to serum killing. The anti-serum ability of MDR strains was more sensitive than that of hypervirulent control strains, but there was no significant difference between the two groups $(P>0.05)$ (Fig. 3).

\section{Biofilm formation assay}

As shown in Fig. 4, the OD values of biofilms formed by MDR strains were ranged from 0.31 to 0.80 , with an average value of $0.58 \pm 0.19$; while the $\mathrm{OD}$ values of biofilms formed by hypervirulent control strains were ranged from 0.06 to 0.39 , with an average value of $0.27 \pm 0.10$. The biofilm formation ability of MDR strains was significantly higher than that of hypervirulent control strains $(P<0.05)$.

\section{Infection model of Galleria mellonella larvae}

As shown in Fig. 5, three MDR strains and three hypervirulent control strains were all time-dependent and concentrationdependent on the lethality of larvae $(P<0.05)$, while the corresponding concentration gradients of MDR strains and hypervirulent control strains had no significant difference in the lethality of larvae (Fig. 5A, B, C, D, E, F). In addition, the lethality of MDR strains and hypervirulent control strains was similar by using $10^{6} \mathrm{CFU} / \mathrm{mL}$ bacterial suspensions to infect larvae, but both were significantly higher than that of the standard strains ATCC 700603 and PBS controls $(P<0.05)(F i g .5 G)$.

\section{Polymerase chain reaction for capsular serotypes and virulence genes}

As shown in Fig. 6, among 12 PLA-causing MDR strains, there were four of K1 serotype, one of K2 serotype, one of K20 serotype and six of non-type. Except for $m a g A$, iro $N$ and $k f u B C$ (ranging from 33.3-50.0\%), all remaining virulence genes were presented in more than half of MDR strains (ranging from 75.0-100\%). The prevalence of $r m p A$ and aerobactin was $83.3 \%$ and $85.3 \%$, respectively. Among 12 hypervirulent control strains, all of them were K1 or K2 serotype, and all virulence genes 
were presented in most strains (ranging from 75.0-100\%) with the exception for iroN (33.3\%). Additionally, the prevalence of numerous virulence genes in MDR strains was not significantly different with hypervirulent control strains.

\section{Multilocus sequence typing}

MLST revealed nine sequence types (ST23, ST11, ST29, ST65, ST86, ST320, ST367, ST420, ST831) in the MDR strains. Among the hypervirulent control strains, the predominant type was ST23 (8/12, 66.7\%), followed by ST65 $(2 / 12,16.7 \%)$ and ST86 (2/12, 16.7\%) (Fig. 6).

\section{Discussion}

MDR K. pneumoniae often infect inpatients with basic diseases and were considered as CKP with a high resistance rate but hypovirulence $[2,12,14]$. It is reported that $K$. pneumoniae isolates from KP-PLA converged hypervirulence and highly resistance that greatly limited the treatment options of KP-PLA, and the condition posed a threat to patients by repeated infections and poor prognosis, but those isolates has not been explored further [16]. To date, little is known about virulence characteristics of these PLA-causing MDR strains. Hence, in the present study, we analyzed the virulence and molecular epidemiology of 12 MDR strains from $163 \mathrm{KP}$-PLA cases in a tertiary teaching hospital from the perspective of clinical characteristics, virulence phenotypes and genotypes. To the best of our knowledge, this study was firstly analyzed the virulence factors of PLA-causing MDR strains.

The general resistant rates were lower in KP-PLA, MDR strains were rare and usually were treated as traditional cKP, and patients infected with MDR strains were more likely to accompany with hepatobiliary diseases than non-MDR ones (Table S1). The general understanding is that these patients are related to hepatobiliary diseases per se and abnormal bilioenteric anatomy, as well as a history of more antibiotic exposure, the hypovirulent cKP with severe resistance rate can easily cause nosocomial infections in these patients, while hypervirulent strains usually infect healthy community residents and are generally fully sensitive to antibacterial drugs. It is worth noting that the high infection-related death rate in patients with PLA of hepatobiliary diseases may be associated with recurrent bacteremia due to MDR bacteria, which can cause uncontrollable infections and more deaths, and clinical symptoms, laboratory examination, and clinical outcomes comparisons between MDR and non-MDR strains were similar (Table S1), suggesting that these MDR isolates may not be the traditional cKP and the acquisition of MDR may not compromise the overall virulence which needs further verification. However, the actual virulence of these MDR strains has not been well evaluated so far.

The results of growth ability suggested there was no fitness cost for the decline in growth ability due to the resistant phenotype. The hypermucoviscosity was considered as a surrogate marker for hvKP [5]. The percentage of hypermucoviscous MDR strains in our study was slightly lower than that of hypervirulent control strains. However, recent studies consider that hypermucoviscosity is unreliable [18]. A polysaccharide capsule can protect $K$. pneumoniae from phagocytosis of immune cells and bactericidal action of complement or antimicrobial peptides and acts as a major virulence factor for hvKP [17]. The results of capsular quantification suggested that the capsular content of PLA-causing MDR strains was between the hypervirulent control strains and the standard strain, which was consistent with the results of string test. When the patients are infected by bacteria, antibodies in human serum specifically will bind to the bacterial epitopes and combine with the complement system to recruit and then kill bacteria for an immune response, and capsular polysaccharide can hinder this process [6]. Although the MDR strains and hypervirulent control strains were all sensitive to serum, the antiserum killing ability of these PLA-causing strains was significantly higher than that of the hypovirulent standard strains, which may be related to the content of capsular polysaccharide. Furthermore, bacteria attach to the surface of host during the infectious process and are coated with polymers such as extracellular polysaccharides and DNA to form biofilms. The physical barrier formed by biofilms can hinder the attack of bacteria by phagocytes and enzymes, which improves the bacterial defenses against host and resistance to antimicrobials [18]. The biofilm formation ability of MDR strains was significantly higher than that of hypervirulent control strains, which may be one of the reasons for the MDR strains to exhibit resistant phenotype. Moreover, $G$. mellonella larvae, as a good model of invertebrate host infection, has been applied to explore the virulence and pathogenicity of PLA-causing MDR K. pneumoniae strains [19]. The virulence in vivo and pathogenicity of MDR strains was comparable with 
hypervirulent control strains. In brief, the PLA-causing MDR K. pneumoniae strains may be hypervirulent with analysis of virulence phenotypes.

Analysis of virulence genotypes can further validate our hypothesis. K. pneumoniae strains are presented in at least 78 capsular serotypes, in which K1 and K2 are related to hvKP, as well as being pathogenic to humans strongly [17, 20]. In the present study, K1 or K2 serotypes accounted for less than half of the PLA-causing MDR strains, while the hypervirulent control strains were all K1 or K2 serotypes. Although K1 or K2 serotypes can regulate the virulence of $K$. pneumoniae, hypervirulence is not unique to these capsular serotypes [21]. In addition, rmpA and aerobactin are the most important genes for hypervirulence [1]. rmpA regulates the synthesis of extracellular polysaccharide capsule to enhance virulence [22-23]. aerobactin is essential for the growth and virulence of $K$. pneumoniae via regulation of iron supply [1]. The high prevalence of $r m p A$ and aerobactin in the MDR strains was slightly lower than that of hypervirulent control strains, reflecting that PLAcausing MDR strains may be combined with hypervirulence from the perspective of virulence genes. Next, $w c a G, m a g A$, and uge genes related to capsule synthesis were also prevalent in PLA-causing MDR strains [24-25]. Moreover, the high prevalence of siderophores genes $y b t A$, entB, and $k f u B C$ in PLA-causing MDR strains suggested that the ability to uptake iron may be equivalent to that of hypervirulent control strains. Furthermore, almost all PLA-causing MDR strains carried fimH (related to type 1 fimbriae), $m r k D$ (related to type 3 fimbriae), and ureA (an a-subunit of the urease, associated with invasion) $[24,26]$, genetically corroborated virulence phenotype results. Therefore, clinicians should be advised to pay more attention to the MDR strains, and also carefully select appropriate managements to treat KP-PLA to reduce bacterial adhesion and colonization.

MLST analysis uncovered the molecular epidemiology of PLA-causing MDR strains. These MDR strains were mainly polyclonal spread, while the clones of hypervirulent control strains were more concentrated, all of which were hypervirulent clones, and ST23 was the predominated type consistenting with previous reports [27]. ST11 is a common carbapenemresistant sequence type in China and even converges hypervirulence, highlighting the great threat of such real superbugs [9, 15]. To the best of knowledge, ST11 carbapenem-resistant hypervirulent strains have not been found in KP-PLA. In this study, there was firstly found one ST11 carbapenem-resistant strain which may be MDR-hypervirulent K. pneumoniae.

\section{Conclusion}

Combining the virulence phenotypes and genotypes, the convergence of hypervirulence and multidrug resistance in PLAcausing MDR K. pneumoniae strains was observed, which might lead to further emergence of a "post-antibiotic" scenario. Importantly, it reminded that clinicians should be highly prudent and cautious in prescribing antibiotics on such KP-PLA patients because of severe drug resistance and take inspection measures timely in view of hypervirulence-induced invasive infections. Moreover, additional studies need to be conducted to elucidate the mechanisms between host, pathogen, and hostpathogen interactions. Further studies can be helpful to raise the awareness of MDR-hvKP and provide effective treatments for KP-PLA patients.

\section{Methods}

\section{Bacterial isolates and antimicrobial susceptibility testing}

During June 1, 2016 to December 31, 2017, a total of 163 KP-PLA cases were collected from the First Affiliated Hospital of Wenzhou Medical University (Wenzhou, China) with an annual admission of more than 160,000 inpatients. The diagnosis of KP-PLA was conducted based on the clinical criteria [7, 28]. Initial strains were isolated from sterile fluid (including pus, blood, and drainage fluid) of KP-PLA patients and identified as K. pneumoniae by matrix-assisted laser desorption/ionization time-offlight mass spectrometry (MALDI-TOF/MS; bioMérieux, Lyons, France). Antimicrobial susceptibility testing of K. pneumoniae isolates was conducted by bioMerieux VITEK-2 (BioMérieux, Marcy-l'Étoile, France) initially. Multidrug resistant strains were defined as non-susceptible to three or more different antimicrobial categories [29]. A total of 12 MDR K. pneumoniae were found in $163 \mathrm{KP}-\mathrm{PLA}$ cases. Meanwhile, an equal number of antimicrobial-susceptible hypervirulent strains were selected as 
the experimental hypervirulent control strains (isolated from healthy, ambulatory patients with KP-PLA and carried both aerobactin and $r m p A$ genes) and the standard strain ATCC 700603 as the hypovirulent standard strain [1, 30].

The minimum inhibitory concentrations (MICs) of ampicillin, aztreonam, ceftriaxone, ceftazidime, cefepime, imipenem, ciprofloxacin, levofloxacin, gentamicin, tobramycin, sulfamethoxazole/trimethoprim, nitrofurantoin and colistin were confirmed by the agar dilution method and microdilution broth method. The results were interpreted by the latest guidelines published by the Clinical and Laboratory Standards Institute (CLSI; Pittsburgh, PA, USA) and the European Committee on Antimicrobial Susceptibility Testing clinical breakpoints (http://www.eucast.org). Escherichia coli ATCC25922 and Pseudomonas aeruginosa ATCC27853 were served as the quality control strains.

\section{Growth curves}

The growth curves of 12 PLA-causing MDR K. pneumoniae isolates were measured by following previous methods [31]. In brief, overnight cultures of selected K. pneumoniae clinical isolates from KP-PLA and K. pneumoniae ATCC 700603 were diluted 1:100 by Luria-Bertani (LB) broth. The cultures were incubated at $37^{\circ} \mathrm{C}$ with constant shaking at $200 \mathrm{rpm}$. Bacteria suspensions were collected at $0,2,4,6,8,10,12,18,24 \mathrm{~h}$ and the absorbance at $600 \mathrm{~nm}$ was determined. Each suspension was measured in triplicates and averages of absorbance values were used for analysis. The growth of PLA-causing MDR $K$. pneumoniae was evaluated by plotting the values of $\mathrm{OD}_{600}$ against time.

\section{String test and quantification of capsule}

The bacterial colonies of $K$. pneumoniae strain on an agar plate were stretched by an inoculation loop. The string test was considered positive when the strain generates a viscous string with a length of $>5 \mathrm{~mm}$, and this strain was also considered hypermucoviscous [30].

Capsule was quantified as described previously with some modifications [10, 32]. Briefly, $500 \mu \mathrm{L}$ of cultured bacteria suspensions were resuspended and adjusted to $10^{8} \mathrm{CFU} / \mathrm{mL}$, and $1.2 \mathrm{~mL}$ sodium tetraborate in sulfuric acid were added in the resuspensions that placed in ice bath and incubated for $5 \mathrm{~min}$ at $100^{\circ} \mathrm{C}$, and then left on ice for $10 \mathrm{~min}$. A $20 \mu \mathrm{L}$ volume of $1.5 \mathrm{mg} / \mathrm{mL} \mathrm{m}$-hydroxyldiphenyl was then added and mixed. After a $5 \mathrm{~min}$ incubation at room temperature, the absorbance at $590 \mathrm{~nm}$ was measured. The glucuronic acid content was determined from a standard curve of glucuronic acid and expressed as $\mu \mathrm{g} / 10^{8} \mathrm{CFU}$. Results were presented as the mean of the data of three independent experiments.

\section{Susceptibility to serum killing}

Serum bactericidal activity was measured using the method as described previously [6]. Bacteria suspensions in nutrient broth were collected during logarithmic phase and adjusted to $10^{6} \mathrm{CFU} / \mathrm{mL}$ of concentration. $25 \mu \mathrm{L}$ of bacteria suspension was added to $75 \mu \mathrm{L}$ of pooled human sera in the tube. Tubes were shaken and incubated for $0,1,2$, or $3 \mathrm{~h}$. An aliquot of each bacterial suspension was removed at the designated time point and diluted corresponding fold by adding Mueller-Hinton broth, and then cultured to determine the number of viable bacteria after exposure to serum. Results were expressed as a percentage of the inoculum and graded, then a strain was considered serum resistant or serum sensitive according to the standards, and each strain was tested at least three times.

\section{Biofilm formation assay}

The biofilm formation assay was measured using the method of Wilksch et al. [33]. Briefly, clinical isolates were grown to logarithmic phase in LB broth and diluted 1:100 with fresh LB broth. A total of $200 \mu \mathrm{L}$ of each dilution were added to a 96-well polystyrene microtiter plate and blank controls were set at the same time, and per strain was set three duplicate wells. Then, the plate was incubated at $37^{\circ} \mathrm{C}$ for $24 \mathrm{~h}$. Planktonic cells were removed, and the wells were washed three times with sterile water, and then stained with $250 \mu \mathrm{L} 0.1 \%$ crystal violet for $10 \mathrm{~min}$ and rinsed three times with sterile water. Stained biofilms were solubilized with $95 \%$ ethanol and quantified by measuring the $\mathrm{OD}_{600}$. Each sample was measured in triplicates, and the averages of absorbance values were used for analysis. 


\section{Infection model of Galleria mellonella larvae}

The model of G. mellonella larvae was carried out on the three PLA-causing MDR isolates (FK3068, FK3228, FK4603) and three control isolates (FK3112, FK3837, FK3914) that were randomly selected and standard strain ATCC 700603 to investigate the virulence and pathogenicity of the strains [34-35]. A serial concentration gradient bacterium suspension of each strain $\left(10^{7}, 10^{6}, 10^{5}, 10^{4} \mathrm{CFU} / \mathrm{mL}\right)$ was prepared in advance. Eight larvae weighing of $200 \mathrm{mg}-250 \mathrm{mg}$ were randomly selected for each strain and each concentration. A $10 \mu \mathrm{L}$ of bacterial suspension was injected into the last left proleg by using a $25 \mu \mathrm{L}$ Hamilton precision syringe. Larvae injected with $10 \mu \mathrm{L}$ phosphate-buffered saline were used as control. And then, the insects were incubated at $37^{\circ} \mathrm{C}$ in the dark and observed after $24 \mathrm{~h}, 48 \mathrm{~h}, 72 \mathrm{~h}$ and $96 \mathrm{~h}$. Larvae were considered dead when they repeatedly failed to respond to physical stimuli.

\section{Polymerase chain reaction (PCR) for capsular serotypes and virulence genes}

Crude genomic DNA was extracted from PLA-causing K. pneumoniae isolates. Subsequently, capsular serotype-specific genes (for serotypes of $\mathrm{K} 1, \mathrm{~K} 2, \mathrm{~K} 5, \mathrm{~K} 20, \mathrm{~K} 54$, and $\mathrm{K} 57$ ) and virulence genes (aerobactin, rmpA, iroN, kfuBC, wcaG, ybtA, magA, fimH, $m r k D$, uge, entB, and ure $A$ ) were amplified by PCR using specific primes as previously described [24, 36-38]. In addition, strains with these genes determined by PCR and DNA sequencing were selected as positive control for the subsequent PCR experiments.

\section{Multilocus sequence typing (MLST)}

In this study, seven housekeeping genes of $K$. pneumoniae (gapA, $m d h$, phoE, tonB, infB, pgi and rpoB) were amplified and sequenced to characterize the genotypes of PLA-causing isolates according to the provided protocols (http://bigsdb.pasteur.fr/klebsiella/klebsiella.html/). The alleles and STs were assigned according to the online database of the Pasteur Institute MLST for K. pneumoniae.

\section{Statistical analysis}

All statistical analyses were performed using SPSS 22.0 software (IBM, Armonk, NY, USA). Continuous variables were expressed as mean values \pm SD or median (25th - 75th percentile), whereas categorical variables were described as the number and percentage of subjects. Comparisons for continuous variables were made using either the Student's $t$ test or the Mann-Whitney Utest, and comparisons for categorical variables using either the Chi-square test or Fisher's exact test. The mortality of G. mellonella were assessed by Kaplan-Meier analysis and log-rank test.

\section{List Of Abbreviations}

PLA: pyogenic liver abscess; MDR: multidrug resistant; MLST: multilocus sequence typing; cKP: classic K. pneumoniae; hvKP: hypervirulent $K$. pneumoniae, KP-PLA: K. pneumoniae induced pyogenic liver abscess; MICs: minimum inhibitory concentrations; PCR: polymerase chain reaction; STs: sequence types

\section{Declarations}

\section{Ethics approval and consent to participate}

This study has been designed in accordance with the Declaration of Helsinki (2013) (https://www.wma.net/policies-

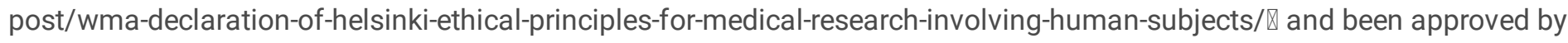
the Ethics Committee in Clinical Research of the First Affiliated Hospital of Wenzhou Medical University (No.2020-070).

\section{Consent for publication}

Not applicable. 
The datasets used and analyzed during the study are available from the corresponding author on reasonable request.

\section{Competing interests}

The authors declare that they have no competing interests.

\section{Funding}

This work was supported by research grants from the National Natural Science Foundation of China (No.81741059 and 81971986) and the Health Department of Zhejiang Province of the People's Republic of China (No. 2019KY098)

\section{Author Contributions}

All authors contributed to data analysis, drafting and revising the article, gave final approval of the version to be published,and agree to be accountable for all aspects of the work.

\section{Acknowledgement}

We thank the National Natural Science Foundation of China (No.81741059 and 81971986) and the Health Department of Zhejiang Province of the People's Republic of China (No. 2019KY098) for providing financial funding.

\section{References}

1. Russo TA, Marr CM. Hypervirulent Klebsiella pneumoniae. Clin Microbiol Rev 2019, 32(3).

2. Siu LK, Yeh KM, Lin JC, Fung CP, Chang FY. Klebsiella pneumoniae liver abscess: a new invasive syndrome. Lancet Infect Dis. 2012;12(11):881-7.

3. Liu YM, Li BB, Zhang YY, Zhang W, Shen H, Li H, Cao B. Clinical and molecular characteristics of emerging hypervirulent Klebsiella pneumoniae bloodstream infections in mainland China. Antimicrob Agents Chemother. 2014;58(9):5379-85.

4. Tsai FC, Huang YT, Chang LY, Wang JT. Pyogenic liver abscess as endemic disease, Taiwan. Emerg Infect Dis. 2008;14(10):1592-600.

5. Shon AS, Bajwa RP, Russo TA. Hypervirulent (hypermucoviscous) Klebsiella pneumoniae: a new and dangerous breed. Virulence. 2013;4(2):107-18.

6. Siu LK, Fung CP, Chang FY, Lee N, Yeh KM, Koh TH, Ip M. Molecular typing and virulence analysis of serotype K1 Klebsiella pneumoniae strains isolated from liver abscess patients and stool samples from noninfectious subjects in Hong Kong, Singapore, and Taiwan. J Clin Microbiol. 2011;49(11):3761-5.

7. Chen J, Zhang M, Chen J, Ning Y, Cai X, Zhang L, Xu H, Guo J. Cryptogenic and non-cryptogenic liver abscess: A retrospective analysis of 178 cases revealed distinct characteristics. J Int Med Res. 2018;46(9):3824-36.

8. Kong H, Yu F, Zhang W, Li X. Clinical and microbiological characteristics of pyogenic liver abscess in a tertiary hospital in East China. Med (Baltim). 2017;96(37):e8050.

9. Gu D, Dong N, Zheng Z, Lin D, Huang M, Wang L, Chan EW, Shu L, Yu J, Zhang R, et al. A fatal outbreak of ST11 carbapenem-resistant hypervirulent Klebsiella pneumoniae in a Chinese hospital: a molecular epidemiological study. Lancet Infect Dis. 2018;18(1):37-46.

10. Yang X, Wai-Chi Chan E, Zhang R, Chen S. A conjugative plasmid that augments virulence in Klebsiella pneumoniae. Nat Microbiol. 2019;4(12):2039-43.

11. Zhang S, Zhang X, Wu Q, Zheng X, Dong G, Fang R, Zhang Y, Cao J, Zhou T. Clinical, microbiological, and molecular epidemiological characteristics of Klebsiella pneumoniaeinduced pyogenic liver abscess in southeastern China. Antimicrob Resist Infect Control. 2019;8:166.

12. Jun JB. Klebsiella pneumoniae Liver Abscess. Infect Chemother. 2018;50(3):210-8. 
13. Liu Y, Liu PP, Wang LH, Wei DD, Wan LG, Zhang W. Capsular Polysaccharide Types and Virulence-Related Traits of Epidemic KPC-Producing Klebsiella pneumoniae Isolates in a Chinese University Hospital. Microb Drug Resist. 2017;23(7):901-7.

14. Ye M, Tu J, Jiang J, Bi Y, You W, Zhang Y, Ren J, Zhu T, Cao Z, Yu Z, et al. Clinical and Genomic Analysis of Liver AbscessCausing Klebsiella pneumoniae Identifies New Liver Abscess-Associated Virulence Genes. Front Cell Infect Microbiol. 2016;6:165.

15. Lee CR, Lee JH, Park KS, Jeon JH, Kim YB, Cha CJ, Jeong BC, Lee SH. Antimicrobial Resistance of Hypervirulent Klebsiella pneumoniae: Epidemiology, Hypervirulence-Associated Determinants, and Resistance Mechanisms. Front Cell Infect Microbiol. 2017;7:483.

16. Su SC, Siu LK, Ma L, Yeh KM, Fung CP, Lin JC, Chang FY. Community-acquired liver abscess caused by serotype K1 Klebsiella pneumoniae with CTX-M-15-type extended-spectrum beta-lactamase. Antimicrob Agents Chemother. 2008;52(2):804-5.

17. Fung CP, Chang FY, Lin JC, Ho DM, Chen CT, Chen JH, Yeh KM, Chen TL, Lin YT, Siu LK. Immune response and pathophysiological features of Klebsiella pneumoniae liver abscesses in an animal model. Lab Invest. 2011;91(7):102939.

18. Flemming HC, Wingender J, Szewzyk U, et al. Biofilms: an emergent form of bacterial life. Nat Rev Microbiol. 2016;14(9):563-75.

19. McLaughlin MM, Advincula MR, Malczynski M, et al. Quantifying the clinical virulence of Klebsiella pneumoniae producing carbapenemase Klebsiella pneumoniae with a Galleria mellonella model and a pilot study to translate to patient outcomes. BMC Infect Dis. 2014;14:31.

20. Podschun R, Ullmann U. Klebsiella spp. as nosocomial pathogens: epidemiology, taxonomy, typing methods, and pathogenicity factors. Clin Microbiol Rev. 1998;11(4):589-603.

21. Yu WL, Ko WC, Cheng KC, Lee CC, Lai CC, Chuang YC. Comparison of prevalence of virulence factors for Klebsiella pneumoniae liver abscesses between isolates with capsular K1/K2 and non-K1/K2 serotypes. Diagn Microbiol Infect Dis. 2008;62(1):1-6.

22. Yu WL, Ko WC, Cheng KC, Lee HC, Ke DS, Lee CC, Fung CP, Chuang YC. Association between $r m p A$ and magA genes and clinical syndromes caused by Klebsiella pneumoniae in Taiwan. Clin Infect Dis. 2006;42(10):1351-8.

23. Paczosa MK, Mecsas J. Klebsiella pneumoniae: Going on the Offense with a Strong Defense. Microbiol Mol Biol Rev. 2016;80(3):629-61.

24. Zhang S, Yang G, Ye Q, Wu Q, Zhang J, Huang Y. Phenotypic and Genotypic Characterization of Klebsiella pneumoniae Isolated From Retail Foods in China. Front Microbiol. 2018;9:289.

25. Yeh KM, Lin JC, Yin FY, Fung CP, Hung HC, Siu LK, Chang FY. Revisiting the importance of virulence determinant magA and its surrounding genes in Klebsiella pneumoniae causing pyogenic liver abscesses: exact role in serotype K1 capsule formation. J Infect Dis. 2010;201(8):1259-67.

26. Struve C, Bojer M, Krogfelt KA. Identification of a conserved chromosomal region encoding Klebsiella pneumoniae type 1 and type 3 fimbriae and assessment of the role of fimbriae in pathogenicity. Infect Immun. 2009;77(11):5016-24.

27. Lam MMC, Wyres KL, Duchene S, Wick RR, Judd LM, Gan YH, Hoh CH, Archuleta S, Molton JS, Kalimuddin S, et al. Population genomics of hypervirulent Klebsiella pneumoniae clonal-group 23 reveals early emergence and rapid global dissemination. Nat Commun. 2018;9(1):2703.

28. Foo NP, Chen KT, Lin HJ, Guo HR. Characteristics of pyogenic liver abscess patients with and without diabetes mellitus. Am J Gastroenterol. 2010;105(2):328-35.

29. Magiorakos AP, Srinivasan A, Carey RB, Carmeli Y, Falagas ME, Giske CG, Harbarth S, Hindler JF, Kahlmeter G, OlssonLiljequist $\mathrm{B}$, et al. Multidrug-resistant, extensively drug-resistant and pandrug-resistant bacteria: an international expert proposal for interim standard definitions for acquired resistance. Clin Microbiol Infect. 2012;18(3):268-81. 
30. Russo TA, Olson R, Fang CT, Stoesser N, Miller M, MacDonald U, Hutson A, Barker JH, La Hoz RM, Johnson JR. Identification of Biomarkers for Differentiation of Hypervirulent Klebsiella pneumoniae from Classical K. pneumoniae. J Clin Microbio/ 2018, 56(9).

31. Palacios M, Broberg CA, Walker KA, Miller VL: A Serendipitous Mutation Reveals the Severe Virulence Defect of a Klebsiella pneumoniae fepB Mutant. mSphere 2017, 2(4).

32. Filisetti-Cozzi TM, Carpita NC. Measurement of uronic acids without interference from neutral sugars. Anal Biochem. 1991;197(1):157-62.

33. Wilksch JJ, Yang J, Clements A, et al. MrkH, a novel c-di-GMP-dependent transcriptional activator, controls Klebsiella pneumoniae biofilm formation by regulating type 3 fimbriae expression. PLoS Pathog. 2011;7(8):e1002204.

34. Zhang X, Zhao Y, Wu Q, Lin J, Fang R, Bi W, Dong G, Li J, Zhang Y, Cao J, et al. Zebrafish and Galleria mellonella: Models to Identify the Subsequent Infection and Evaluate the Immunological Differences in Different Klebsiella pneumoniae Intestinal Colonization Strains. Front Microbiol. 2019;10:2750.

35. Insua JL, Llobet E, Moranta D, et al. Modeling Klebsiella pneumoniae pathogenesis by infection of the wax moth Galleria mellonella. Infect Immun. 2013;81(10):3552-65.

36. Candan ED, Aksoz N. Klebsiella pneumoniae: characteristics of carbapenem resistance and virulence factors. Acta Biochim Pol. 2015;62(4):867-74.

37. Luo Y, Wang Y, Ye L, Yang J. Molecular epidemiology and virulence factors of pyogenic liver abscess causing Klebsiella pneumoniae in China. Clin Microbiol Infect. 2014;20(11):0818-24.

38. Wasfi R, Elkhatib WF, Ashour HM. Molecular typing and virulence analysis of multidrug resistant Klebsiella pneumoniae clinical isolates recovered from Egyptian hospitals. Sci Rep. 2016;6:38929.

\section{Figures}

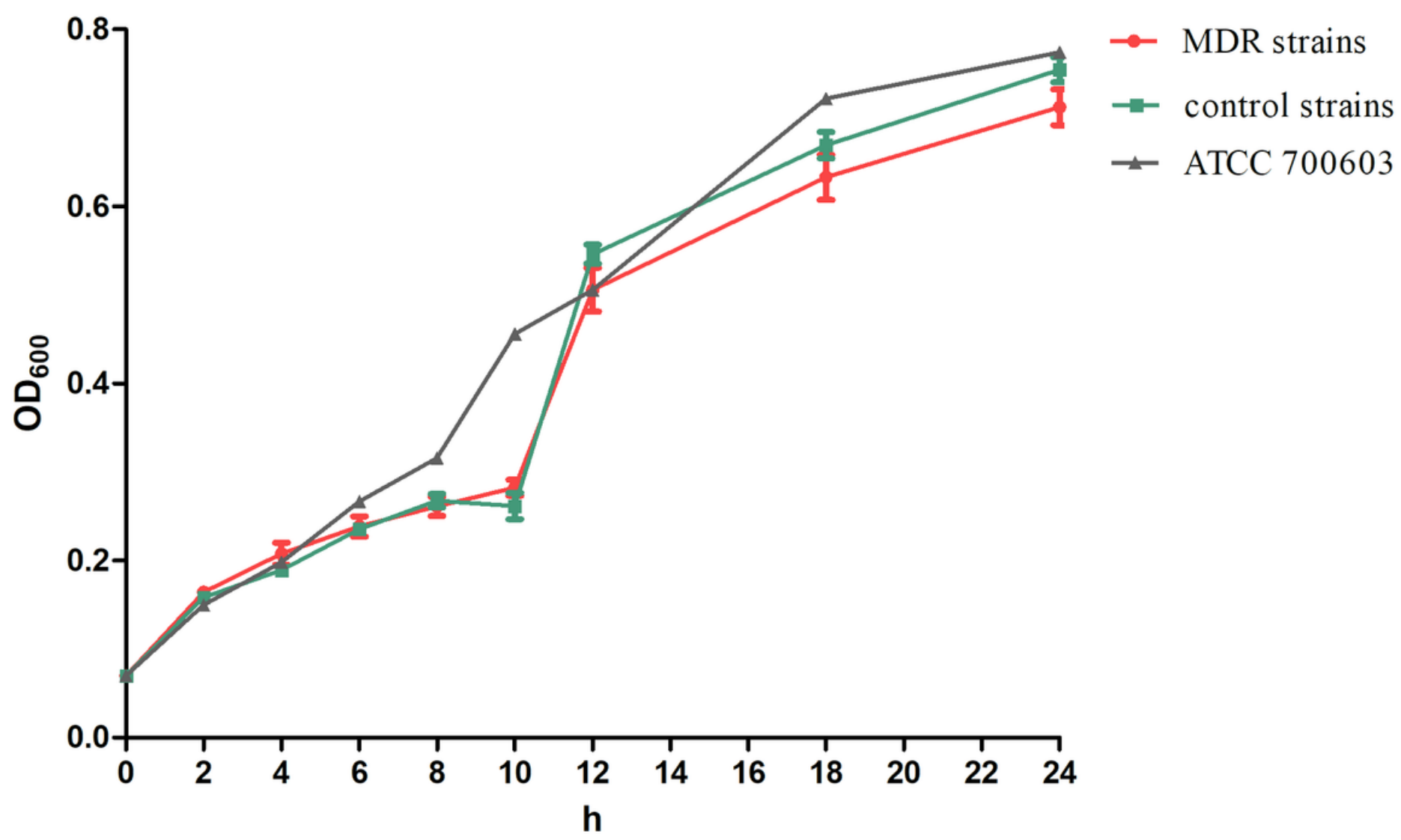


Figure 1

The growth curves of PLA-causing multidrug resistant K. pneumoniae strains

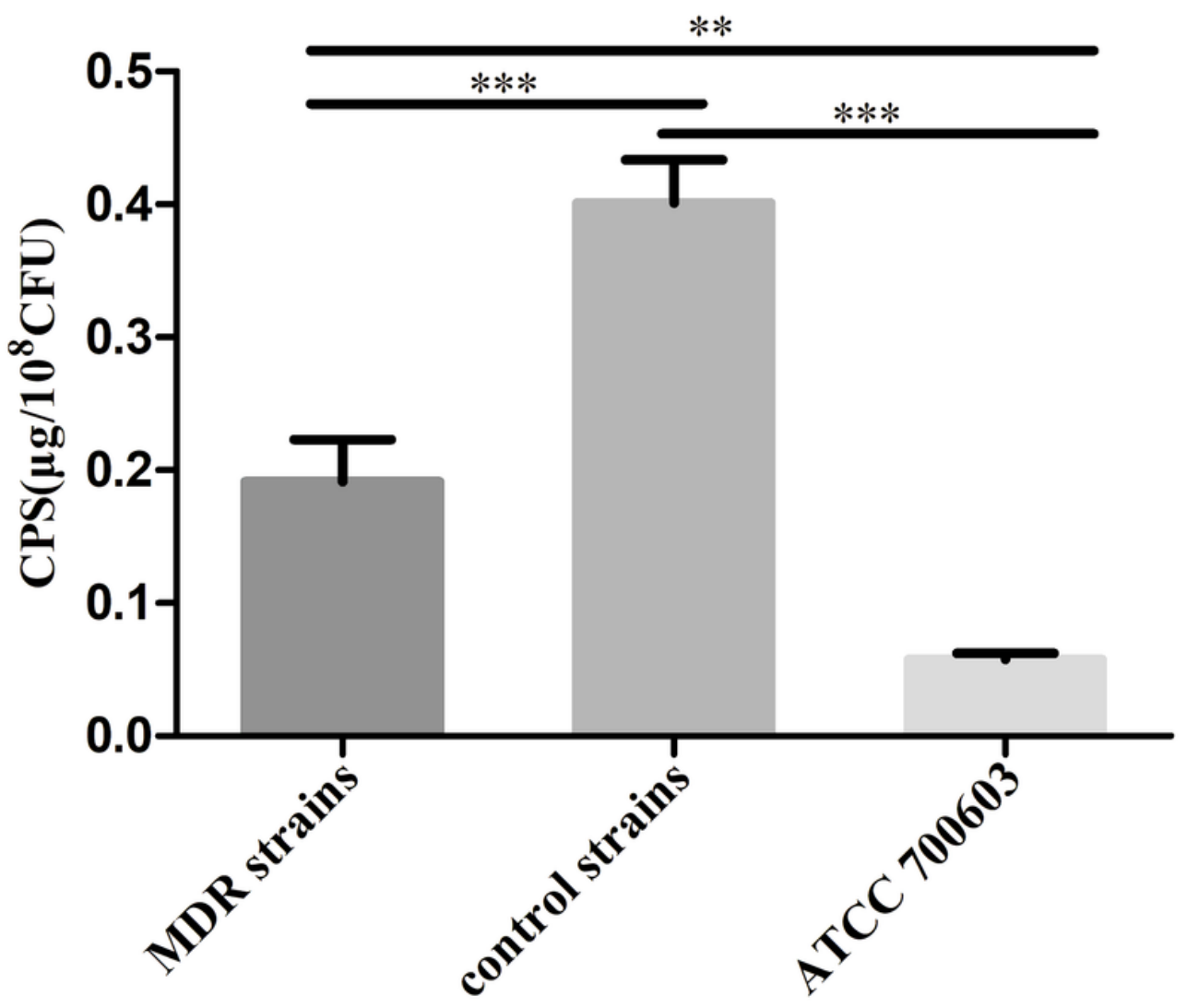

\section{MDR strains} control strains ATCC 700603

Figure 2

The capsular quantification of PLA-causing multidrug resistant $\mathrm{K}$. pneumoniae strains 


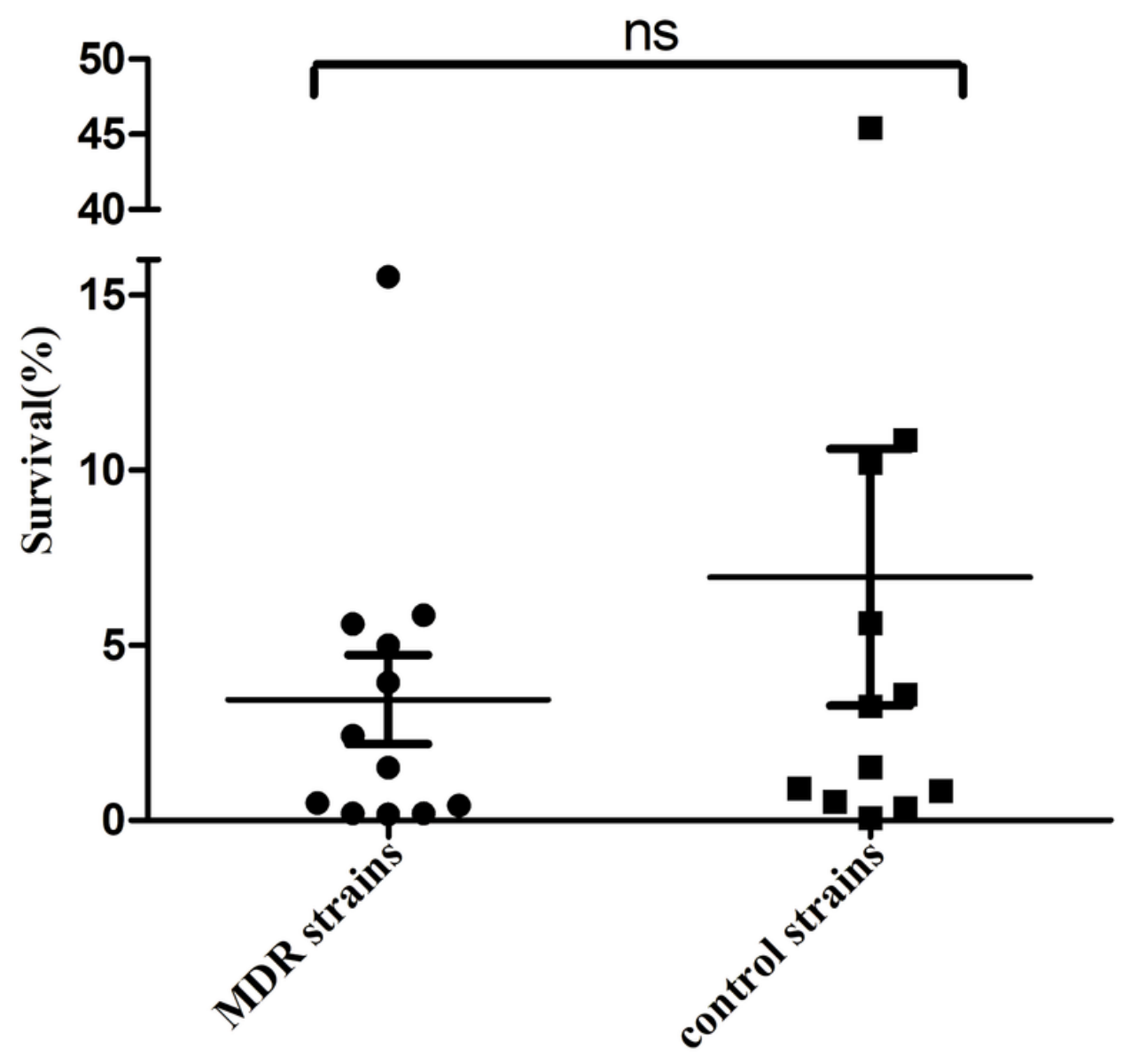

- MDR strains

- control strains

\section{Figure 3}

The anti-serum ability of PLA-causing multidrug resistant K. pneumoniae strains 


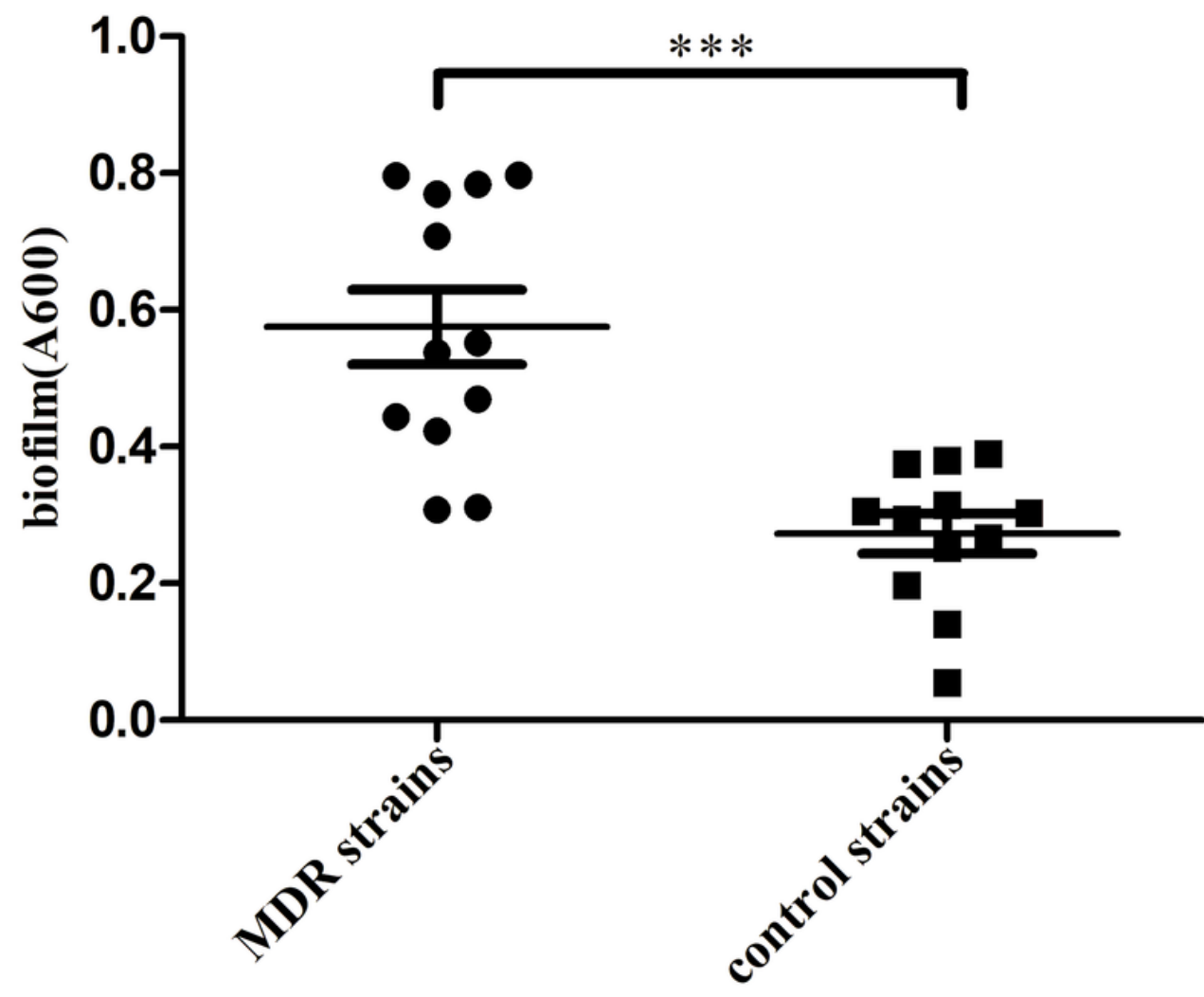

Figure 4

The biofilm formation ability of PLA-causing multidrug resistant $\mathrm{K}$. pneumoniae strains 

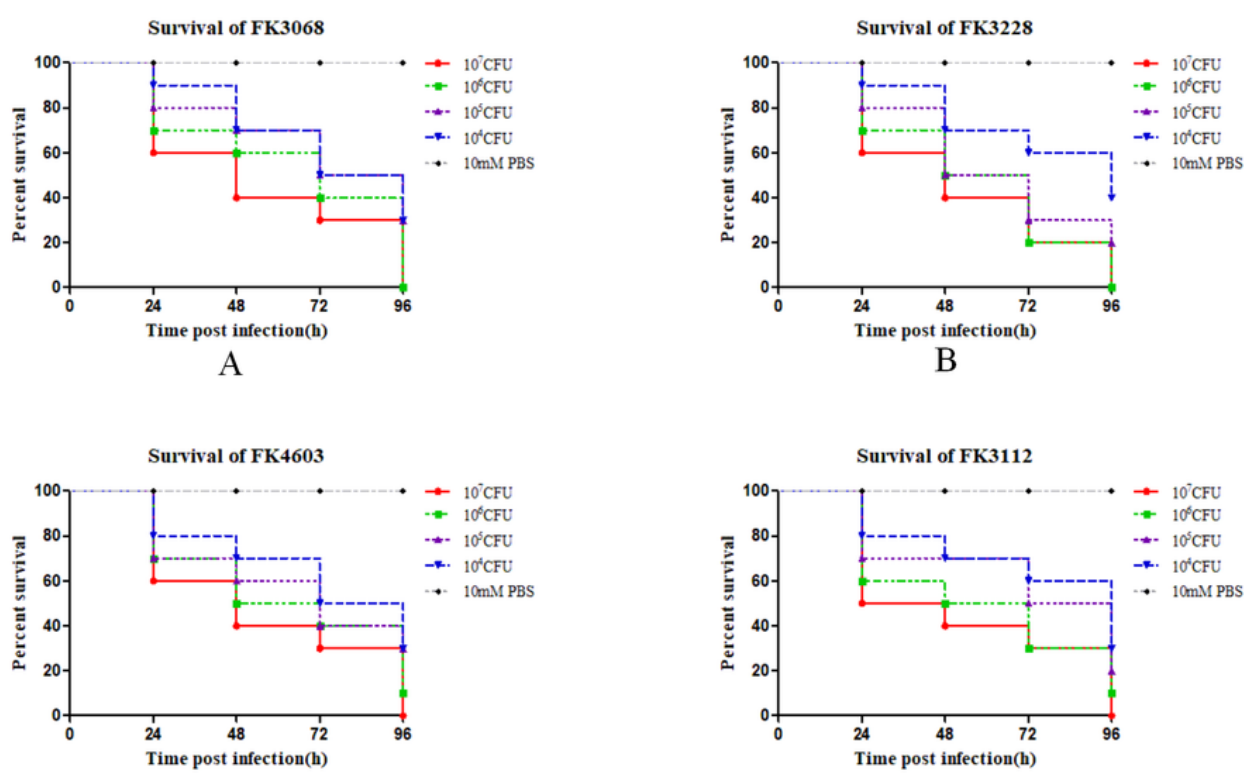

$\mathrm{C}$

D
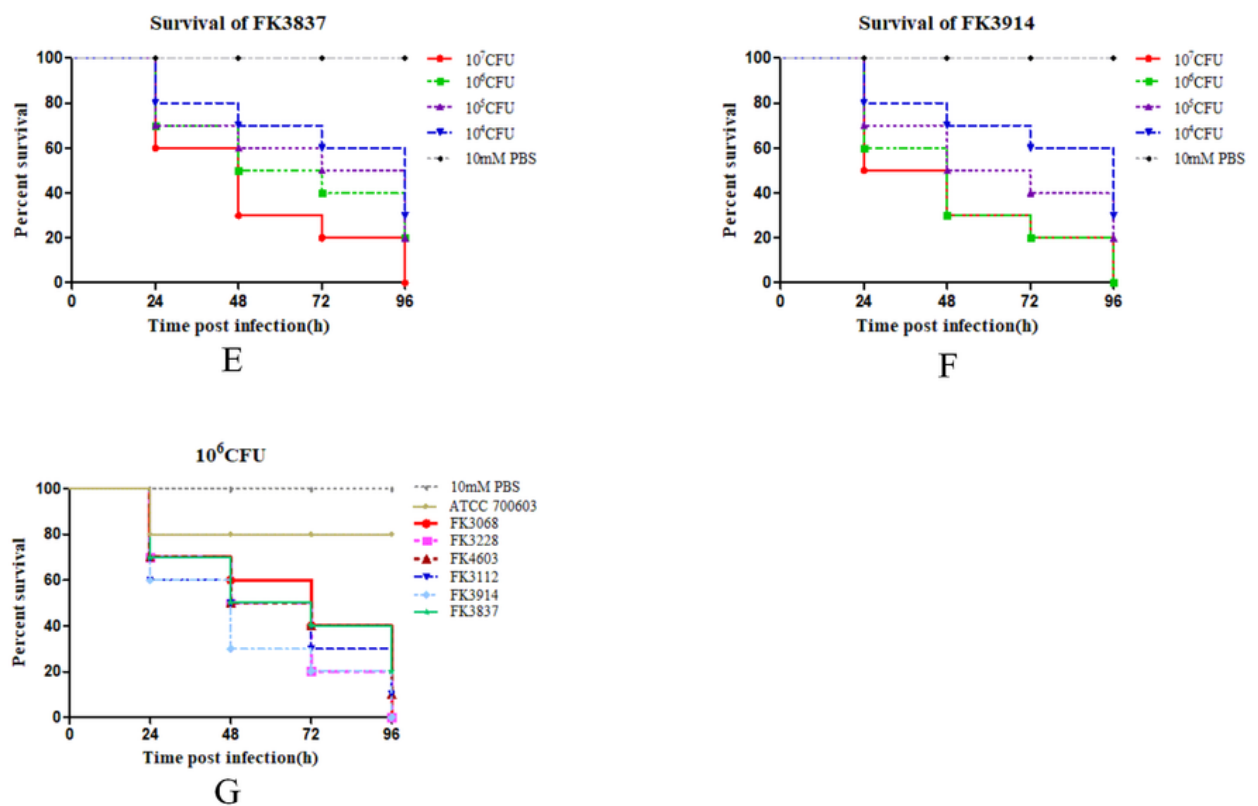

Figure 5

Survival curves of K. pneumoniae infection model of Galleria mellonella larvae 


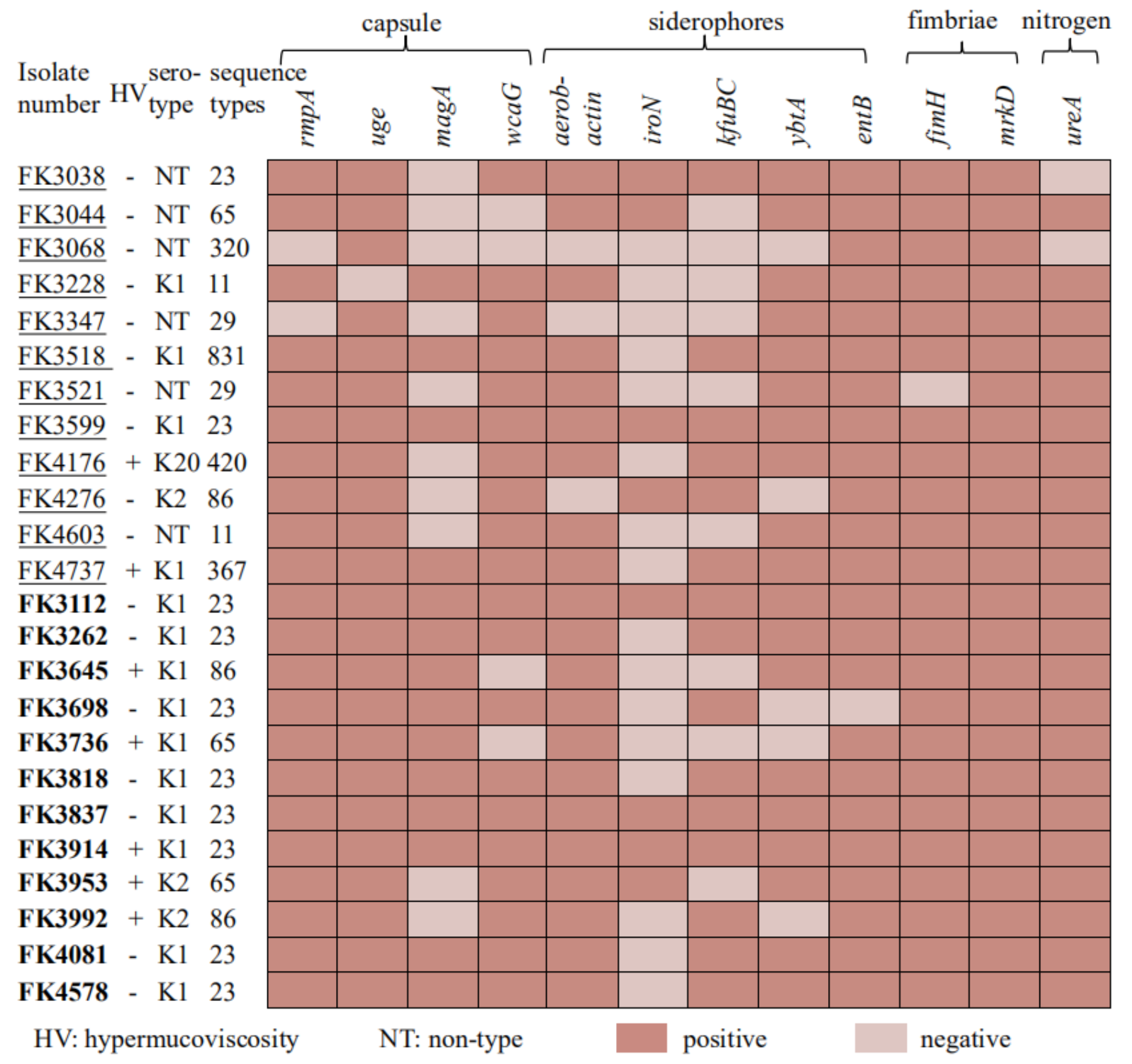

Figure 6

Genotype map of PLA-causing multidrug resistant and hypervirulent control strains

\section{Supplementary Files}

This is a list of supplementary files associated with this preprint. Click to download.

- Tables1.docx 